University of Wollongong

Research Online

Faculty of Engineering - Papers (Archive)

Faculty of Engineering and Information

Sciences

$1-1-2007$

\title{
Multi-stacked artificial muscle actuator based on synthetic elastomer
}

Huu Chuc Nguyen

Sungkyunkwan University

JongKil Park

Sungkyunkwan University

Vu Thuy Doan

Sungkyunkwan University

Hyun-Seok Kim

Sungkyunkwan University

Ja Choon Koo

Sungkyunkwan University

See next page for additional authors

Follow this and additional works at: https://ro.uow.edu.au/engpapers

Part of the Engineering Commons

https://ro.uow.edu.au/engpapers/4898

\section{Recommended Citation}

Nguyen, Huu Chuc; Park, JongKil; Doan, Vu Thuy; Kim, Hyun-Seok; Koo, Ja Choon; Lee, Youngkwan; Nam, Jae-do; and Choi, Hyouk R.: Multi-stacked artificial muscle actuator based on synthetic elastomer 2007, 771-776.

https://ro.uow.edu.au/engpapers/4898

Research Online is the open access institutional repository for the University of Wollongong. For further information contact the UOW Library: research-pubs@uow.edu.au 


\section{Authors}

Huu Chuc Nguyen, JongKil Park, Vu Thuy Doan, Hyun-Seok Kim, Ja Choon Koo, Youngkwan Lee, Jae-do Nam, and Hyouk R. Choi 


\title{
Multi-stacked Artificial Muscle Actuator Based on Synthetic Elastomer
}

\author{
Nguyen Huu Chuc, Jong Kil Park, Doan Vu Thuy, Hyun Seok Kim, Ja Choon Koo, \\ Youngkwan Lee, Jae-do Nam, Hyouk Ryeol Choi
}

\begin{abstract}
In this paper, we present a new artificial muscle actuator for the robotic applications, called multi-stacked actuator. This actuator is made from a new material, named synthetic elastomer previously developed by ourselves[1]. The synthetic elastomer displays enhanced performance in terms of electrical as well as mechanical properties, which can be adjusted depending on its applications. The actuator is composed of the synthetic elastomer sheet coated with compliant electrodes on the both sides, connecting disks, spring, and rigid frame. This novel design enables the actuator to generate the large strain as well as the large force. Experimental works are performed to evaluate the actuation performance and the effectiveness of the actuator is validated.
\end{abstract}

\section{INTRODUCTION}

During last decades, a great deal of researches on electroactive polymer actuators (EAP) have been conducted and remarkable progresses have been made. Among them, dielectric elastomer actuators are one of the most prospective because of its large deformation and force, and cost effectiveness[2]. There are numerous dielectric elastomers available commercially such as silicone, polyurethane, acrylic elastomer etc.[3]. However, they have critical drawbacks because of their characteristic properties, typically dielectric constants, and elastic modulus, can not be under prospective modification according to the requirements[2]. Dielectric elastomers currently available do not permit the change of their material properties. In order to get an actuator practically applicable, it is necessary to develop new materials whose properties can be easily adjusted. In the previous report, an elastomeric material, called synthetic elastomer was presented [1]. It has many advantages, in particular it allows us to modify the properties of the material according to the requirements, which results in the actuation characteristics. Thus, its applications can be extended from macro to nano scale.

Actuators made from the dielectric elastomer have been applied in the robots, micro devices, micro air vehicles, etc. So far, various configurations of actuators have been proposed such as planar, tube, roll extender, diaphragm and bender, etc. [4], [5], [6], [7]. The roll or tube actuators have advantage of large deformation and high force. However,

This work was supported by the 21st Century Frontier R\&D Programs funded by the Ministry of Commerce, Industry and Energy

N. H. Chuc, J. K. Park, , D. V. Thuy, H. S. Kim, J. C. Koo, Y. K. Lee, J. D. Nam, H. R. Choi are with School of Mechanical Engineering, Sungkyunkwan University, Suwon, Kyunggi-do, Korea

Y. K. Lee, J. D. Nam are with School of Applied Chemistry and Chemical Engineering, Sungkyunkwan University, Suwon, Kyunggi-do, Korea

All correspondences are to be sent to Prof. Choi at hrchoieskku.ac.kr
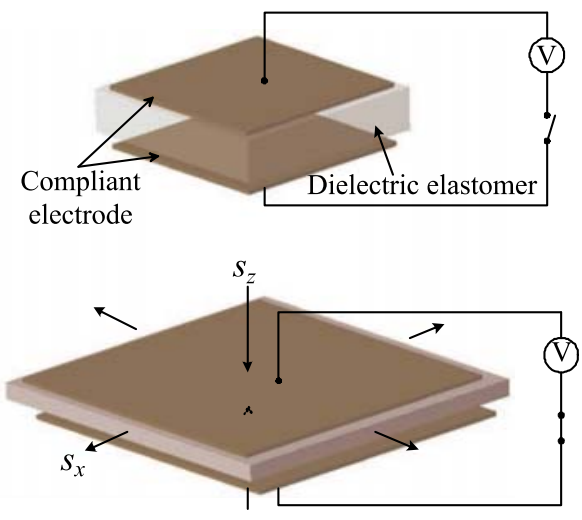

Fig. 1. The principle of dielectric elastomer

most of the robotic actuators developed up to now employ extremely large prestrain (over 300\%) to amplify the strain as well as the output force[4], [5].

Recent investigations note that the prestrain has influence on the mechanical as well as the electrical properties of the actuator[13]. As a critical drawback, in particular, its performance is time-dependent so that, the performance is getting worse after several cycles of actuation, because the prestrain is reduced continuously as the time goes on, which makes the actuation force and displacement decrease. Recently, Choi et. al reported an actuation method without prestrain and applied in driving the artificial annelid robot[8]. The folded dielectric elastomer by Carpi et. al. that is equivalent to multilayer stack actuators, can produce a large deformation[9]. Moreover, an automatic fabrication technology have been developed to provide electrostatic multi layer stack actuator applied for Braille diplay[10]. The advantage of these multilayer stack actuators is to have a deformation originated directly from the Maxwell stress. As shown in Fig. 1, when the dielectric elastomer is unconstrained and a driving voltage is applied, the strain $s_{z}$ along the thickness direction is the result of Maxwell stress, meanwhile the strain $s_{x}$ along the lateral direction can be calculated from the incompressibility condition as follows.

$$
s_{z}=2 s_{x}
$$

According to Eq. (1), the strain along the thickness direction is twice than the one along lateral direction. Consequently, the method using the deformation that comes from the principle of Maxwell stress is simple and allows us a stable and larger deformation. Nevertheless, there are still several issues to be investigated, for example, design, fabrication, 
driving circuitry etc., which are mostly focused on improving the displacement or force of the actuator[9].

In this paper, a new actuator producing linear motion, called multi-stacked actuator, is proposed. It is made from the synthetic elastomer and a novel design of the actuator for robotic applications is proposed. The challenges are how to realize the actuation characteristics according to the requirements, which can be achieved by adjusting the material properties and the design of the actuator. In addition, the proposed actuator does not need any prestrain to amplify the displacement or force. It just includes multiple layers of synthetic elastomer layers that electrode layers is connected in parallel to minimize the resistance of electrode. The details about the proposed actuator will be described in the following.

This paper is organized as follows. In the first, the synthetic elastomer is described briefly in section II. In the section III, the structure of the multi stacked actuator is outlined in details. Also, the process and fabrication of the proposed actuator are introduced in section IV. The experimental results will be discussed and evaluated in section V. Finally, conclusions with future works are given in section VI.

\section{SYNTHETIC ELASTOMER}

To begin with the study of the actuator, it is necessary to consider significant parameters influencing on the overall performance of actuation. According to the fundamental physics of the dielectric elastomer, the principle operation is based on the electromechanical transduction of a parallel two plate capacitor. It generates a electrostatic force, called Maxwell stress. The Maxwell stress is expressed as

$$
\sigma_{e}=-\varepsilon_{o} \varepsilon_{r} E^{2}=-\varepsilon_{o} \varepsilon_{r}\left(\frac{V}{t}\right)^{2}
$$
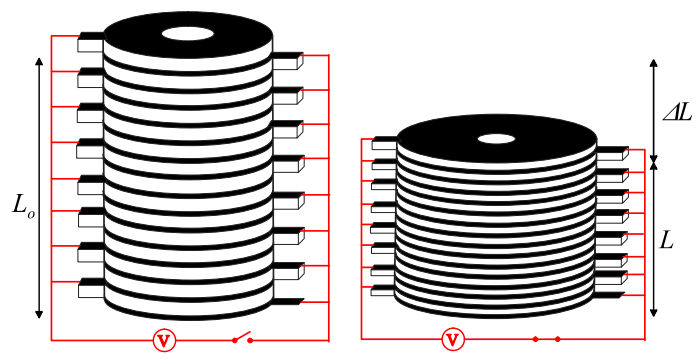

Fig. 2. The operation principle of multi-stacked actuator

where $\varepsilon_{o}$, and $\varepsilon_{r}$ denote the permittivity of the free space and the relative permittivity of the elastomer, respectively. $E$, $V$, and $t$ represent the electric field, the applied voltage, and the thickness of the dielectric elastomer, respectively. As the dielectric constant of the elastomer becomes larger, the more electromechanical stress and energy density are obtained with a much smaller input voltage. It should be noted that the dielectric constant $\varepsilon_{r}$ is one of the most important material properties of actuation.
The important factors of the dielectric elastomer are represented with dielectric constant, breakdown strength, elastic modulus, and stress relaxation, and viscoelasticity [1]. Among dielectric elastomers, Acrylonitrile Butadiene Rubber (NBR) has an excellent characteristic adjusting material properties to the requirements above. NBR is the result of copolymerization of acrylonitrile(ACN) and butadiene rubber(BR). The $\mathrm{ACN}$ is a polar rubber with high dielectric constant and good elongation as well as adequate resilience, tensile and compression set. The content of ACN is a primary factor characterizing NBR. The ACN level, because of its polarity, determines the basic mechanical properties, such as oil and solvent resistance, as well as electrical properties like dielectric constant and breakdown voltage[11], [12]. Additionally, the mechanical properties of NBR can be adjusted by vulcanization conditions. Moreover, we can adjust the material properties of the NBR by changing the recipe of the additives which play an important role in the manufacturing process. In other words, material properties can be changed a lot by controlling the contents of the ACN, additives, and vulcanization conditions. This is the reason why the NBR can be considered as a promising candidate for dielectric elastomer actuator and the details can be referred to [1], [2]

\section{DESIGN}

The proposed actuator is designed to be directly driven by Maxwell stress without using prestretching. In addition it produces a relative large displacement(up to $15 \%$ of the effective length of the actuator) because the novel synthetic elastomer is utilized. Its fundamental principle of operation is as shown in Fig. 2. When the voltage is applied between two electrode layers, Maxwell stress is produced, and thus the dielectric elastomer is compressed along the axial direction. The compression of each layer results the lateral expansion of the actuator because of the incompressibility of the polymer. Consequently, the deformation of the multi stacked actuator is the sum of the deformation of each layer, and the total deformation is expressed as

$$
\Delta L=\sum_{i=1}^{N} \Delta L_{i}=N \Delta L_{i}
$$

The principles are simple but its performance is quite different depending on the design of the actuator. In particular the geometry of the actuator unit has large influence on. In this paper, two types of design, that is circular actuator and rectangular one are introduced.

As illustrated in Fig. 3, the basic unit of the circular actuator is composed of the synthetic elastomer and compliant electrode layers put on both sides of the elastomer. The actuator is just built up by stacking the circular actuator units and having electrical connections among the basic units. Electrical connectors are placed to face the opposite sides as shown in Fig. 3 in order to avoid the electrical shortage. In addition, the inactive region not covered with electrode layers needs to be kept as small as possible because it obstructs the movement of the active region that covered by the electrode. It is important to minimize the inactive 


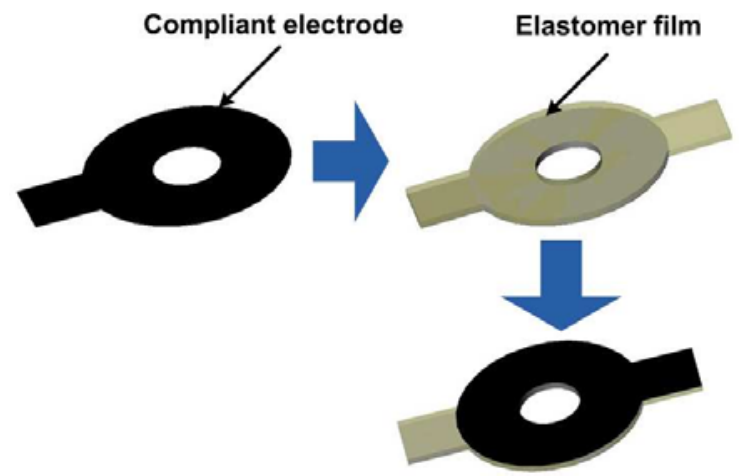

Fig. 3. Design of actuator unit

boundary, though it cannot be removed completely due to the electrical shortage. The circular actuator is easy to fabricate and simple, but the displacement of the actuator can be reduced because the inactive region entirely surrounds the active one. The hole inside the actuator is introduced to help break the stress balance inside the actuator and increase the displacement. The basic actuator units are stacked to make a bundle of the actuator and then, the bundles of actuators are stacked again passing through a shaft as illustrated in Fig. 4. In addition, connecting disks are inserted to do without obstruction between the boundaries of the bundles and realize a large deformation. In addition a spring is inserted to make the stacked actuator compressed tightly. It is used to support the compression of the actuator when they are applied with power source.

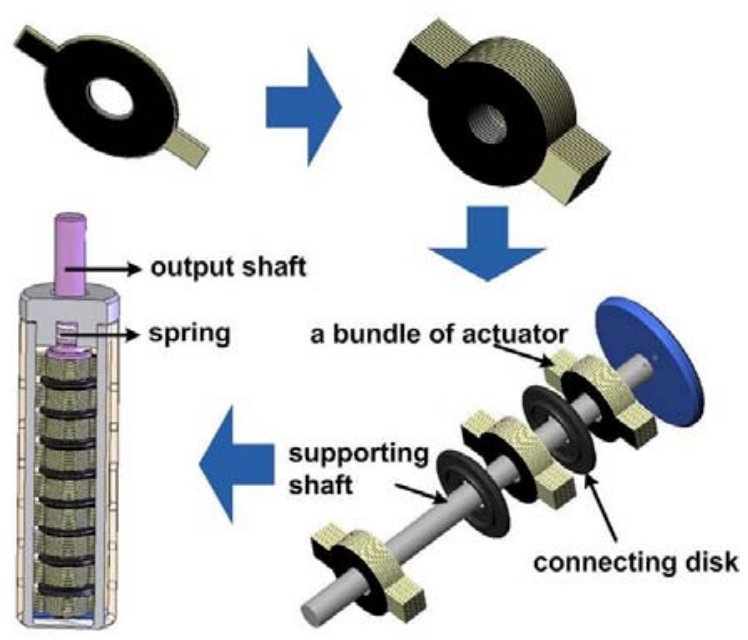

Fig. 4. Design of circular actuator

As mentioned above, the inactive boundary obstructing the deformation of the actuator, needs to be kept as small as possible. The second prototype, called the rectangular actuator, is designed to cope with this problem. It is designed to minimize the inactive boundary by folding as shown in Fig. 5. After being folded, the actuator has one free direction without the boundary, which helps the actuator generate the improved deformation than the previous ones.
Fundamentally, the rectangular actuator has difference from Carpi's work because separate connection among the actuator units or driving can be possible[9]. Except that, most features are similar to the circular actuator as shown in Fig. 6.

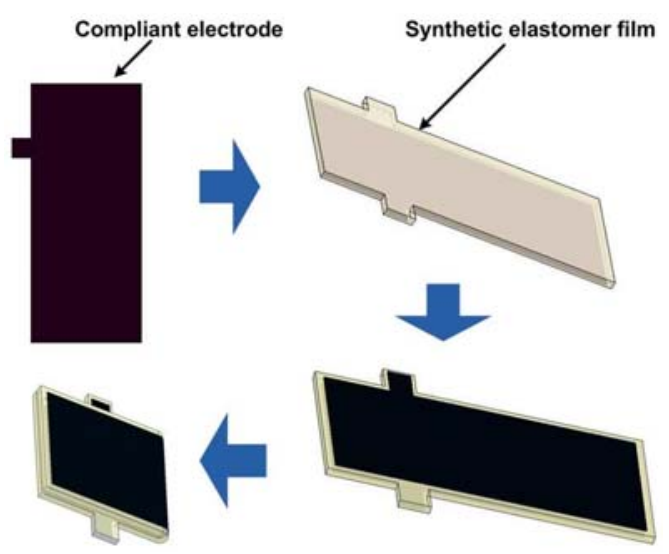

Fig. 5. Design of the rectangular actuator unit
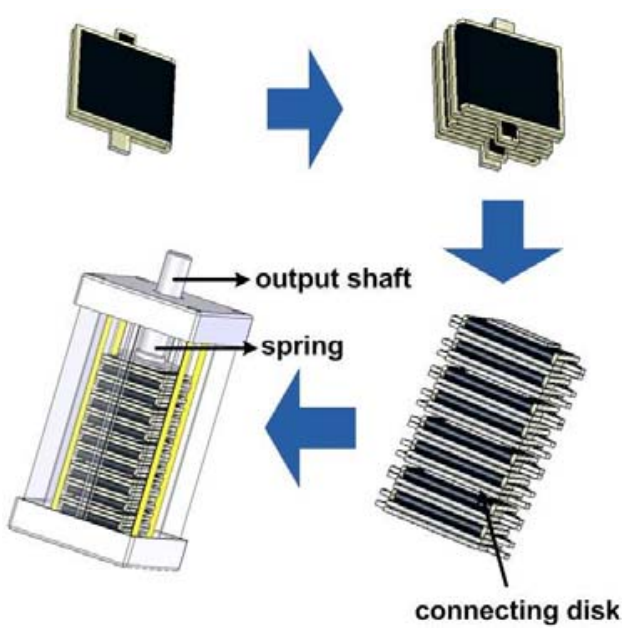

Fig. 6. Design of rectangular actuator

\section{FABRICATION}

The fabrication process is as illustrated in Fig. 7. First, the synthetic rubber is resolved with a solvent. Secondly, the additives to make the material softer and to cross-link the material are mixed with the determined composition. Selection of additives are very much important because it plays major role on tuning the material properties. That is, elastic modulus, dielectric constant, stress relaxation and breakdown voltage etc. are determined by the additives, typically such as sulfur, DO (DimethylOxide), DOP(Diotyl phthalate) etc. In the third, the synthetic rubber is dispensed by using the dispensing machine, and the synthetic elastomer is produced after cross-linking in the vacuum oven at a high temperature. Here, the solution of the electrode is produced by mixing carbon powder and synthetic elastomer and the solution is dispensed similarly. 


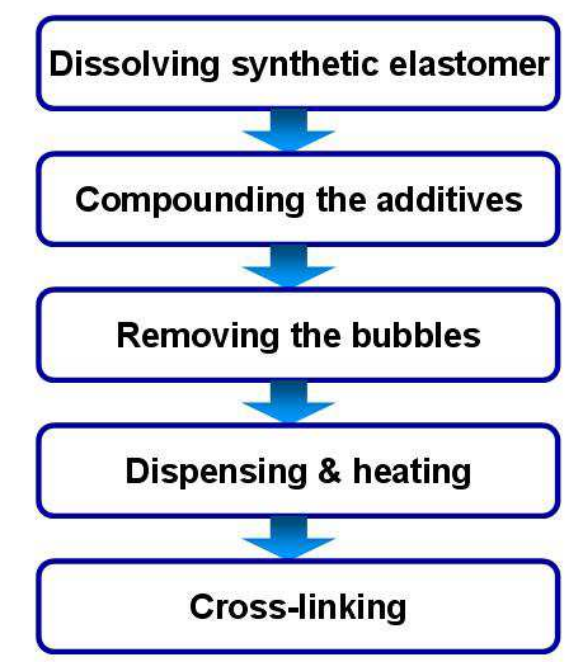

Fig. 7. Manufacturing process

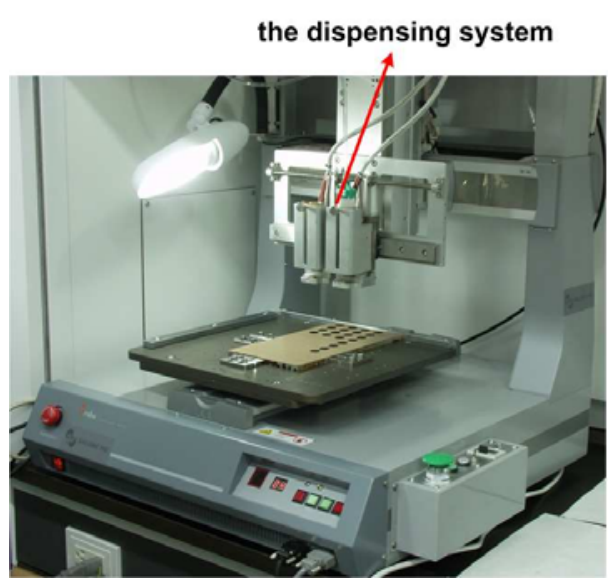

Fig. 8. The robotic system for automatic manufacturing

The actuator is fabricated automatically by using a dedicated robot system as shown in Fig. 8. The details about this machine is described in [15]. The robot dispenses the synthetic elastomer according to the sources assembled. Typically, it is not dispensed at a time, but very thin synthetic elastomer is dispensed repetitively and stacked. It is a very important to have the uniform layer of the synthetic elastomer. Accuracy of the dispensing system is around $0.3 \mathrm{~mm}$ in the lateral direction. Minimum thickness of dispensing layer is $15 \mu \mathrm{m}$. The thickness of the electrode layer is about $20 \mu \mathrm{m}$, and that of a synthetic elastomer is typically about $60 \mu \mathrm{m}$, respectively. All the actuators are produced on the teflon sheet. The housings are made of engineering plastic and the bearing is used to reduce the friction between the cap and the output shaft. Two actuator prototypes manufactured are as shown in Fig. 9.

\section{ANALYSIS AND EXPERIMENTS}

\section{A. Analysis}

The performance of the actuator has been analyzed by ANSYS and the results are as shown in Fig. 10. In the

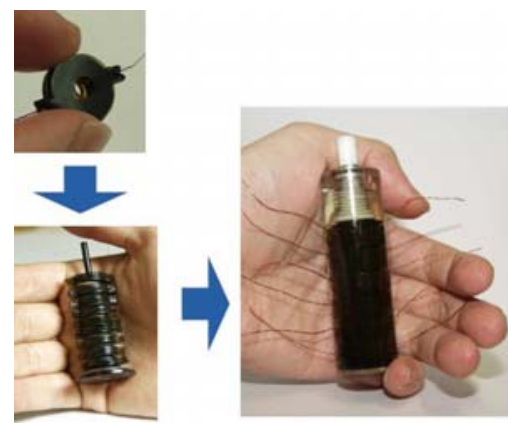

(a) Annulus actuator

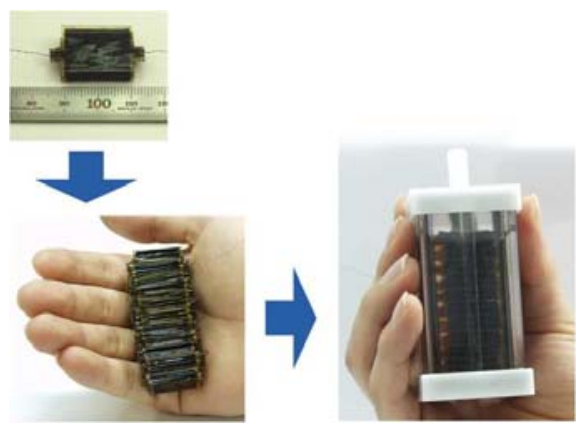

(b) Rectangular actuator

Fig. 9. The prototype of the actuators.

analysis, the size of the inactive boundary in the circular actuator is $0.5 \mathrm{~mm}$ both inside and outside boundaries. Whereas the rectangular actuator has the boundary of $0.5 \mathrm{~mm}$ in three sides and no boundary in the other side. As noted in the analysis, the rectangular actuator has larger deformation than the annulus one. It is because the rectangular one has a free boundary, though the circular one does not. The free boundary alleviates the deformation of the actuator.

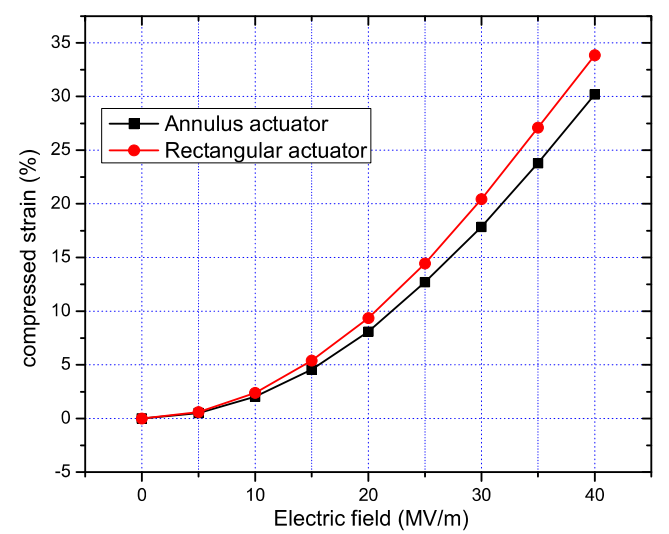

Fig. 10. Results of analysis and comparison between two actuators

\section{B. Experiments}

In the experiments, several aspects of the actuator were tested to evaluate the actuator. The experimental setup is as depicted in Fig. 11. It was designed for measuring the motion of the actuator and the load can be put on 


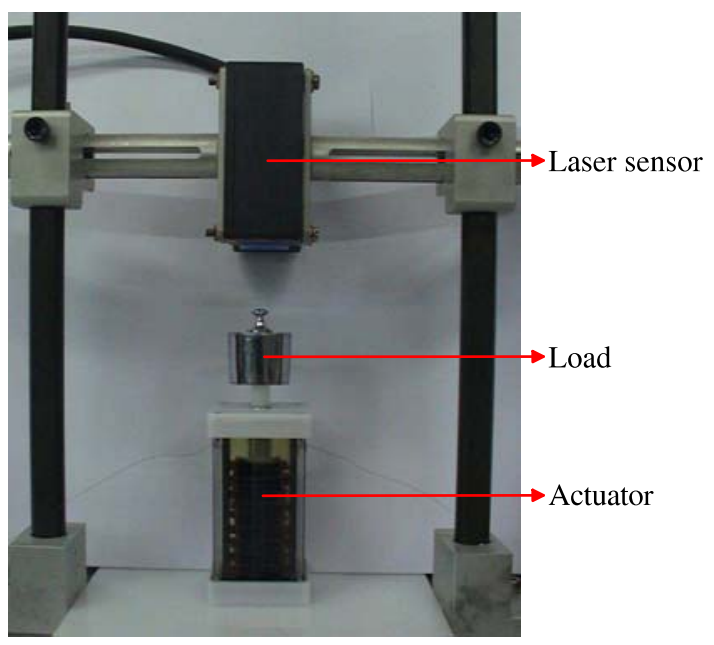

Fig. 11. Experimental setup for testing the actuator

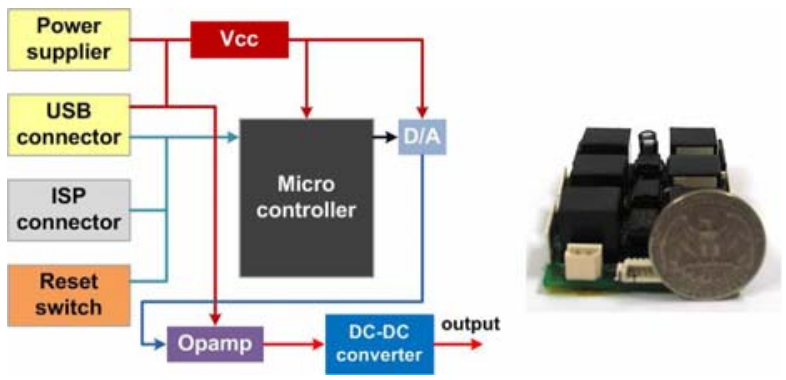

Fig. 12. The portable driving circuit

the output shaft of the actuator to verify the output force. The displacement of the actuator was measured with a laser displacement sensor (LK-081 Keyence) and the data was collected via a Universal Serial Bus (USB) to the personal computer. A high-voltage amplifier (Trek-10/10B) with a function generator and a voltage supplier was installed to supply sinusoidal driving voltages with variable frequencies. Additionally, a miniature driving circuitry was made as shown in Fig. 12. Input of the circuit is just $5 \mathrm{~V}$ and the DC-DC converter amplifies the applied voltage by 1000 times to supply the high voltage to the actuator up to $5 \mathrm{kV}$. It was developed to demonstrate the possibility in robotic applications.

In the first experiment, the displacements of the actuators were measured with different loads: $50 g, 100 g$, and $150 g$, respectively. The frequency of the driving input was set $1 \mathrm{~Hz}$ of the sinusoidal wave. The experimental results are shown in Figs. 13 and 14. It notes that the displacement of the actuator decreased when the mass of the load is increased. As discussed in Fig.10 the deformation of the rectangular actuator is better than the circular one. A unit of actuator, in case of rectangular one, originally had almost deformations of $10 \%$ at $3.5 \mathrm{KV}$, but when it is stacked, the total ratio of the deformation is reduced compared to those of a unit of the actuator. It may be caused by the mechanical interferences of the actuator such as friction, etc.

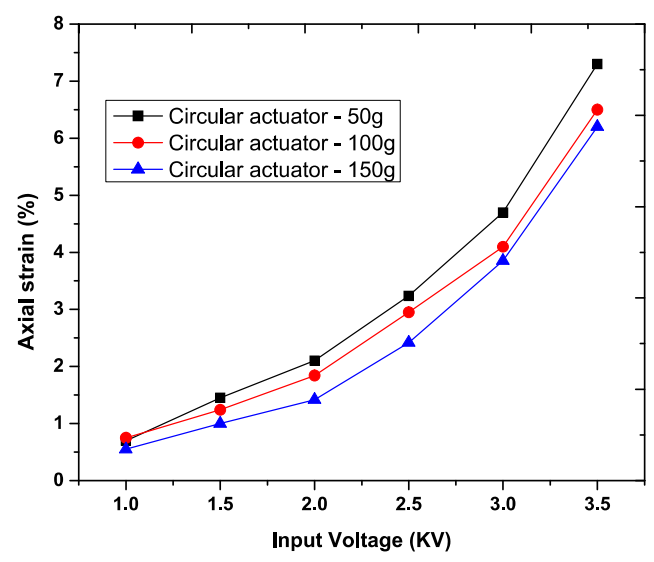

Fig. 13. Strain of the circular actuator

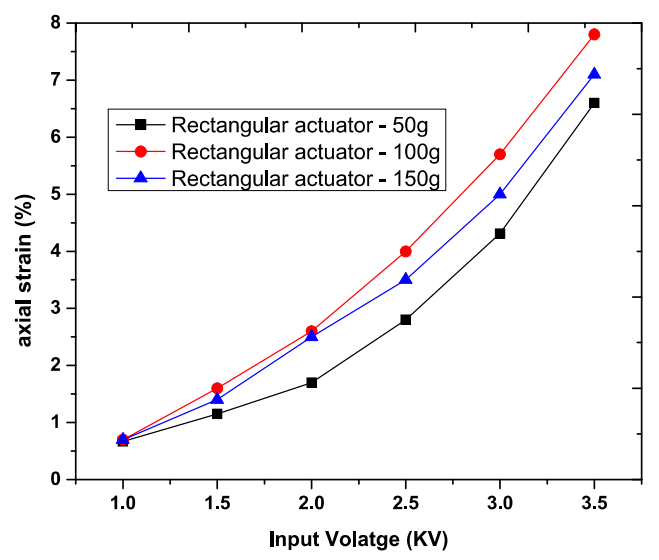

Fig. 14. Strain of the rectangular actuator

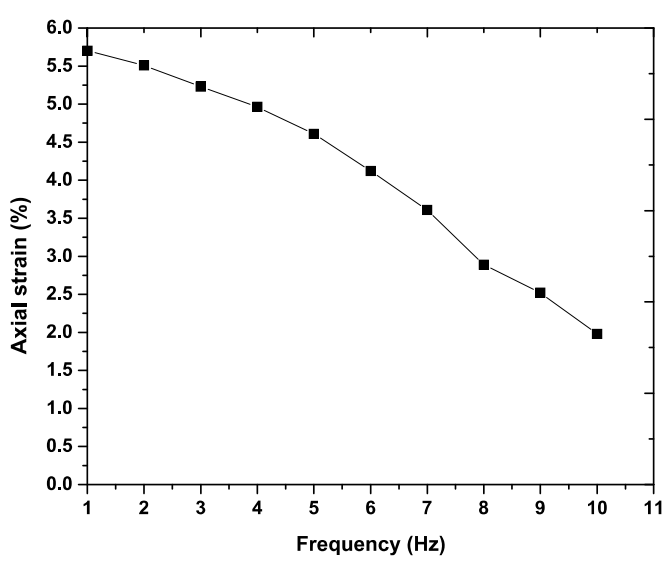

Fig. 15. Frequency response of the rectangular actuator 
The frequency response was measured, too. The applied voltage was kept at $3 K V$ and an external load was also kept at $100 \mathrm{~g}$. The frequency was swept from $1 \mathrm{~Hz}$ to $10 \mathrm{~Hz}$. As shown in Fig. 15, the bandwidth of the system remains around several hertz. It is just the result of the test for the actuator only, though it appears a little bit low for robotic applications. It can be improved by introducing dedicated circuitry as presented in the previous reports[14].

\section{CONCLUSIONS}

In this paper, a multi-stacked artificial muscle actuator based on the novel synthetic elastomer was presented. We demonstrated that the rectangular actuator has better performance than the circular one due to the different mechanical structure in relation to the boundary. The proposed actuator has advantages for controlling each layer independently and providing macro forces as well as displacements suitable for robotic applications. In the future, various control approaches will be studied to apply for controlling the developed actuator.

\section{ACKNOWLEDGMENT}

This research was performed for the Intelligent Robotics Development, one of the 21 st Century Frontier R\&D Programs funded by the Ministry of Commerce, Industry and Energy.

\section{REFERENCES}

[1] K. M. Jung, J. H. Lee, M. S. Cho, J. C. Koo, J. D. Nam, Y. K. Lee, H. R. Choi, "Development of enhanced synthetic elastomer for the energy efficient polymer actuators," Smart Materials and Structures, Vol. 16, pp. S288-S294, 2007.

[2] K. J. Kim, and S. Tadokoro, Ed., "Electroactive Polymer for Robotic Applications," Springer, 2007.

[3] R. Pelrine, R. Kornbluh, Q. Pei, J. Joseph, "High-speed Electrically Actuated Elastomer with Strain Greater Than $100 \%$," Science, Vol. 287, pp. 836-839, 2000.

[4] Q. Pei, R. Pelrine, S. Stanford, R. Kornbluh, M. Rosenthal, "Electroelastomer rolls and their application for biomimetic walking robots," Synth. Met., Vol. 135-136, pp. 129-131, 2003.

[5] Q. Pei, M. Rosenthal, S. Stanford, H. Prahlad, R. Pelrine, "Multipledegrees-of-freedom electroelastomer roll actuators," Smart Materials and Structures, Vol. 13, pp. N86-N92, 2004.

[6] H. R. Choi, K. M. Jung, S. M. Ryew, J. D. Nam, J. W. Jeon, J. C Koo, K. Tanie, "Biomimetic Soft Actuator: Design, Modelling, Control and Applications," IEEE/ASME Transaction on Mechatronics, Vol. 10, No. 5, pp. $581-593,2005$

[7] M. Y. Jung, K. M. Jung, N. H. Chuc, I. M. Koo, J. C. Koo, Y. K Lee, J. D. Nam, H. R. Choi, "Fabrication and characterization of linear motion dielectric elastomer actuators," EAPAD, Vol. 6168, pp. $616824-$ $1-7,2006$

[8] K. M. Jung, J. C. Koo, J. D. Nam, Y. K. Lee, H. R. Choi, ”Artificial annelid robot driven by soft actuators," J. Bioinspiration and Biomimetics, Vol. 2, pp. S42-S49, 2007.

[9] F. Carpi, C. Salaris, D. Rossi, "Folded dielectric elastomer actuators," Smart Materials and Structures, Vol. 16, pp. S300-S305, 2007.

[10] H. F. Shlaak, M. Jungmann, M. Matysek, P. Lotz, ”Novel Multilayer Electrostatic Solid-State Actuators with Elastic Dielectric," EAPAD, Vol. 5759, pp. 121-131, 2005.

[11] D. Mackey, A. H. Jorgensen, "Elastomers, Synthetic(Nitrile Rubber)," Kirk-Othmer Concise Encyclopedia of Chemical Technology, 1999

[12] W. Hofmann, "NITRILE RUBBER," Rubber Chemistry and Technology, 1964

[13] H. R. Choi, K. M. Jung, N. H. Chuc, M. Y. Jung, I. M. Koo, J. C Koo, J. H. Lee, J. H. Lee, J. D. Nam, M. S. Cho, Y. K. Lee, "Effects of Prestrain on Behavior of Dielectric Elastomer Actuator," EAPAD, Vol. 5759, pp. 283-291, 2005.
[14] I. M. Koo, K. M. Jung, J. C. Koo, J. H. Lee, J. H. Lee, J. D. Nam, M. S. Cho, Y. K. Lee, H. R. Choi, "Wearable Tactile Display Based on Soft Actuator," IEEE International Conference of Robotics and Automation, pp. 2220-2225 , 2006

[15] The DTR series manual at www.dasatech.com 